\title{
The Influence of Abrasive Size on High-Pressure Chemical Mechanical Polishing of Sapphire Wafer
}

\author{
Chuljin Park', Hyoungjae Kim', Sangjik Lee', and Haedo Jeong2,, \\ 1 Korea Institute of Industrial Technology, 30, Gwahaksandan 1-ro 60beon-gil, Gangseo-gu, Busan, 618-230, South Korea \\ 2 Graduate School of Mechanical Engineering, Pusan National University, 2, Busandaehak-ro 63beon-gil, Geumjeong-gu, Busan, 609-836, South Korea \\ \# Corresponding Author / E-mail: hdjeong@pusan.ac.kr, TEL: +82-51-510-2463, FAX: +82-51-510-8442
}

KEYWORDS: Chemical mechanical polishing (CMP), High-Pressure, Abrasive size, Removal amount, Friction, Sapphire wafer

\begin{abstract}
Demand for sapphire wafer has increased with growth of LED market. Chemical mechanical polishing (CMP) comprises a large part of wafering cost since the CMP process requires approximately 3-6 hours. For longer polishing times, the cost of consumables (COC) in CMP increases the total wafering cost; hence, considerable efforts have been made to decrease the polishing time of sapphire wafers to reduce the COC. There are two main approaches to reduce polishing time: controlling the chemical factor and adjusting the mechanical factor. Controlling the chemical factor is a common approach to manipulating the removal rate and roughness. However, it is hard to control the chemical factor. Instead, this study investigates the effects of various mechanical factors. This paper focuses on the effect of high-pressure CMP on the material removal performance; the maximum applied pressure is $\sim 800 \mathrm{~g} / \mathrm{cm}^{2}$. The removal rate increases linearly with gradual increase of CMP pressure to $800 \mathrm{~g} / \mathrm{cm}^{2}$. Finally, the effect of high pressure on the removal rate of, and frictional force on, sapphire wafer during CMP using different sized abrasives is investigated; the effect of the abrasives on the removal rate is likewise analyzed at different pressures.
\end{abstract}

Manuscript received: July 7, 2014 / Revised: September 15, 2014 / Accepted: September 30, 2014

\section{NOMENCLATURE}

$\mathrm{V}=$ the total removal amount

$\mathrm{k}=$ process variables

$\Delta \mathrm{A}=$ contact area of an abrasive with substrate

$1=$ the moving distance of an abrasive on a surface

$\mathrm{n}=$ the number of active abrasives

\section{Introduction}

LEDs are one of the most sustainable lighting sources because they have much longer lifespans and they consume less energy than other lighting sources. The application of LEDs is rapidly expanding to broad industrial fields such as monitors, lamps, televisions, mobile phones, etc. As a result, it is of interest to reduce the manufacturing cost in order to lower LED prices.

$\mathrm{GaN}$ is a very important thin film material, which produces blue light in an LED. A sapphire substrate is required to grow the GaN thin film because its lattice constant is well matched to that of $\mathrm{GaN} .^{1-3}$
Manufacturing the sapphire substrate is difficult since sapphire is hard, brittle and chemically stable. ${ }^{4-6}$ Wire saw, lapping, Diamond Mechanical Polishing (DMP), Chemical mechanical polishing (CMP) are proceeded sequentially as wafering manufacturing process. Sapphire ingot is cut to substrate by wire saw, thickness of substrate is controlled by lapping, thickness deviation is minimized by DMP, and surface condition is improved by CMP. CMP is the most difficult among the sapphire wafering processes because it requires approximately 3-6 hours of processing time to remove subsurface damage and to achieve atomic level roughness. This long polishing time for CMP strongly affects the total wafering cost. ${ }^{7}$ Therefore, manufacturers are trying to reduce the polishing time by increasing the removal rate. Most approaches have been chemical, such as adjusting the $\mathrm{pH}$, adding chemical agents, etc.; these chemical approaches have limitations, however, due to the chemical stability of sapphire. On the other hand, the removal rate could be increased more effectively by changing the mechanical factors using Preston's equation. The main mechanical factors in sapphire wafer CMP are the pressure, the relative velocity, and the abrasive used.

C-plane sapphire substrates react with strong alkaline and acid base slurries. However, these slurries are not generally used because they are very dangerous for CMP. Furthermore, the other plane-oriented 
sapphire substrates do not chemically react with these slurries ${ }^{7}$ so it is difficult to find $\mathrm{pH}$, against chemicals for CMP of sapphire; currently, additional chemical agents have been developed hardly.

Pressure is one of the easiest mechanical factors to control in sapphire wafer CMP. In general condition, the removal rate is nearly $1 \mathrm{um} / \mathrm{h}$. The removal rate increases linearly with pressure according to Preston's equation; the surface condition after polishing is also good enough to use the wafer as a substrate for growing GaN.

The removal rate and surface condition of sapphire wafer can be improved by adjusting the size of the abrasive used in CMP. Lu has previously examined how particle size influences the removal rate and surface condition of copper and tantalum $;{ }^{8}$ it was found that the most important factor is that the particles be monodisperse and round. Building on this, the present study finds that the removal rate increases linearly with the abrasive size, except if the particles are not monodisperse and round, in which case the removal rate is not stable.

Similarly, the relative velocity affects the removal rate. Up to a certain threshold, increasing the relative velocity increases the removal rate, while past this threshold, the removal rate begins to decrease. ${ }^{9}$ The optimum relative velocity depends on the pressure and the polishing slurry viscosity. As has been previously noted,$^{10}$ the decrease in the removal rate above the optimum relative velocity may be caused by a hydrodynamic effect that results in a poorer mechanical contact between the sapphire and the polishing pad.

Optimizing the mechanical factors is needed in order to improve the efficiency of sapphire wafer CMP. Drawing from previous studies, the two most important factors are the abrasive size and the pressure. Understanding the characteristics of each parameter is necessary to find the combination effect between pressure and particle size in sapphire CMP. The present study investigates the effects of these two mechanical factors on the removal rate and the frictional force.

\section{Experimental Conditions}

\subsection{Removal Test}

Since various mechanical factors significantly change the CMP characteristics, ${ }^{8-14}$ it is prudent to investigate how the pressure, and the size and number density of the abrasive affect the removal rate. In the present study, the removal rate is evaluated with abrasive sizes of $40 \mathrm{~nm}$, $72 \mathrm{~nm}$, and $82.5 \mathrm{~nm}$, under pressures of $400 \mathrm{~g} / \mathrm{cm}^{2}$ and $800 \mathrm{~g} / \mathrm{cm}^{2}$. Table 1 shows the conditions used in this study for sapphire wafer CMP; every

Table 1 Experimental conditions of CMP test

\begin{tabular}{cc}
\hline Parameters & Conditions \\
\hline CMP machine & Desktop polisher \\
\hline Slurry & $\begin{array}{c}\text { Colloidal silica slurry } \\
\text { (COMPOL, FUJIMI Corporation, } \mathrm{pH} 9.8)\end{array}$ \\
\hline Abrasive size & $40 \mathrm{~nm}, 72 \mathrm{~nm}, 82.5 \mathrm{~nm}$ \\
\hline Pressure & $400 \mathrm{~g} / \mathrm{cm}^{2}, 800 \mathrm{~g} / \mathrm{cm}^{2}$ \\
\hline CMP conditions & Platen rotation : $120 \mathrm{rpm}$ \\
\hline Slurry flow rate & $400 \mathrm{ml} / \mathrm{min}$ \\
\hline Polishing pad & Nonwoven polyurethane pad \\
\hline Polishing time & 30 minutes \\
\hline Wafer & Epi-ready 2 inch sapphire wafer
\end{tabular}

experiment was repeated five times to improve accuracy.

\subsection{Friction Test}

Brittle sapphire is likely to be removed in the ductile mode due to nano-sized abrasives: when abrasives contact the surface and an external force is applied, a force is generated between the surface and abrasives. ${ }^{15,16}$ This is a frictional force. Furthermore, this mechanism is a principal source of friction and can explain why the removal rate increases with the frictional force. Fig. 1 shows a schematic of the experimental setup of CMP and friction force, which was also used to conduct the friction test: the frictional force can be measured via minute movements of the head.

As the number of abrasives increases, the contact between the surface and the abrasives also increases. Similarly, as the size of the abrasives increases, the contact area between the surface and the abrasives increases. Consequently, increasing the size and number of the abrasives will cause the frictional force to increase.

In the friction experiment, the variation of the frictional force with changes in the pressure and abrasive size was evaluated using the same number of abrasives. The experimental conditions for the friction experiment are listed in Table 2, with the other unlisted conditions being the same as those listed in Table 1. Since the parameters in Table 2 are suitable for measuring the frictional force, the time, slurry flow rate and rotation speed were all decreased and a 4-inch wafer was used. These experiments were also repeated five times to improve accuracy.

\subsection{Abrasive}

The slurry used in this experiment had a $\mathrm{pH}$ of 9.8 and was composed of colloidal silica. Fig. 2 shows the distributions of three different slurries with mean abrasive sizes of $40 \mathrm{~nm}, 72 \mathrm{~nm}$ and $82.5 \mathrm{~nm}$. Fig. 3 shows transmission electron microscope (TEM) images of the abrasives in the three slurries. The abrasive sizes are measured

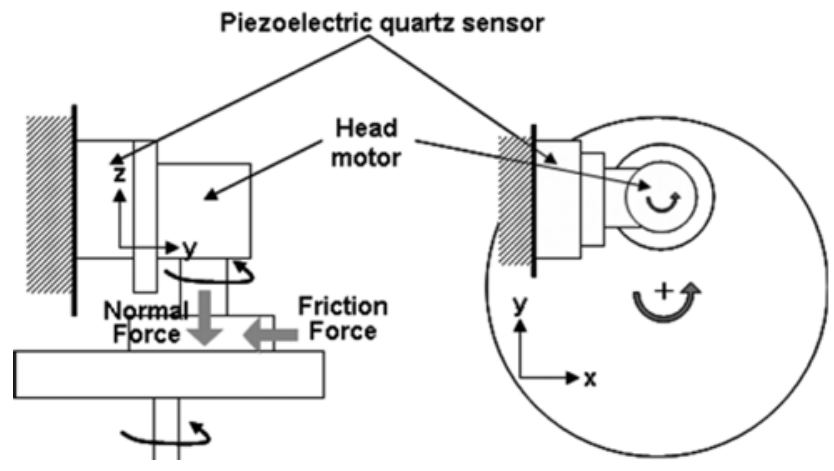

Fig. 1 Schematic diagram of CMP friction test

Table 2 Experimental conditions of friction test

\begin{tabular}{cc}
\hline Parameters & Conditions \\
\hline CMP machine & POLI-400 $(\mathrm{GnP}$ Technology, Korea $)$ \\
\hline Pressure & $200 \mathrm{~g} / \mathrm{cm}^{2}, 400 \mathrm{~g} / \mathrm{cm}^{2}, 600 \mathrm{~g} / \mathrm{cm}^{2}, 800 \mathrm{~g} / \mathrm{cm}^{2}$ \\
\hline CMP conditions & $\begin{array}{c}\text { Platen rotation }: 93 \mathrm{rpm} \\
\text { Head rotation }: 87 \mathrm{rpm}\end{array}$ \\
\hline Slurry flow rate & $250 \mathrm{ml} / \mathrm{min}$ \\
\hline Polishing time & $3 \mathrm{minutes}$ \\
\hline Wafer & Epi-ready 4 inch sapphire wafer \\
\hline
\end{tabular}


with the TEM, while the distribution is measured using a Particle Size Distribution (PSD, DLS-7000, Otsuka Electronics Co., Ltd.) device. The PSD device what we used is not precise enough to measure the abrasive size (it usually measures larger than actual size) but rather it is used to determine whether the abrasive is monodisperse. The particle sizes measured with the TEM were consistent with the quoted information from the COMPOL (FUJIMI Corporation). The slurries all had the same number and volume distribution of abrasives. The abrasives at sizes of $40 \mathrm{~nm}$ and $72 \mathrm{~nm}$ were almost round and regular, and were monodisperse, as confirmed by the TEM and the PSD device. However, the slurry with abrasives at a size of $82.5 \mathrm{~nm}$ con tained considerable variation in particle size, which was not very well captured in the abrasive size distribution analysis. Furthermore, the large size abrasives had quite irregular shapes, as seen in Fig. 3.
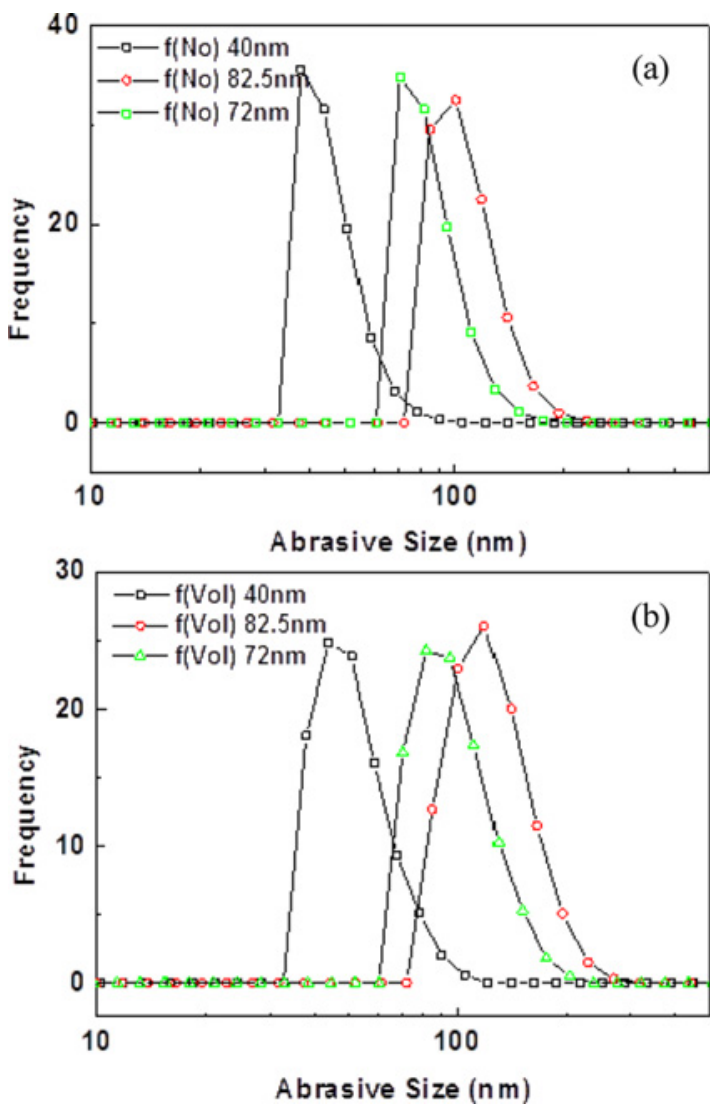

Fig. 2 Distrivution of silica slurry: (a) Number and (b) Volume

\section{Experimental Results and Discussion}

\subsection{Effect of Abrasive Size}

In order to investigate the effect of the abrasive size on the removal rate and the frictional force, each of the slurries were diluted with de-ionized water to achieve slurries with the same particle number concentration. The number of abrasives was estimated using the density and particle size. The number of particles per unit volume for the $82.5 \mathrm{~nm}$ slurry was the lowest of the three at $7.9 \times 10^{14} \mathrm{~cm}^{-3}$. Therefore, the remaining two slurries were diluted to the same particle number density.

Fig. 4 shows that the frictional force is linearly proportional to the applied pressure and that the removal rate increases with increasing abrasive size. Note that the $82.5 \mathrm{~nm}$ slurry has a relatively low removal rate and high frictional force, which is likely due to the irregular shapes and
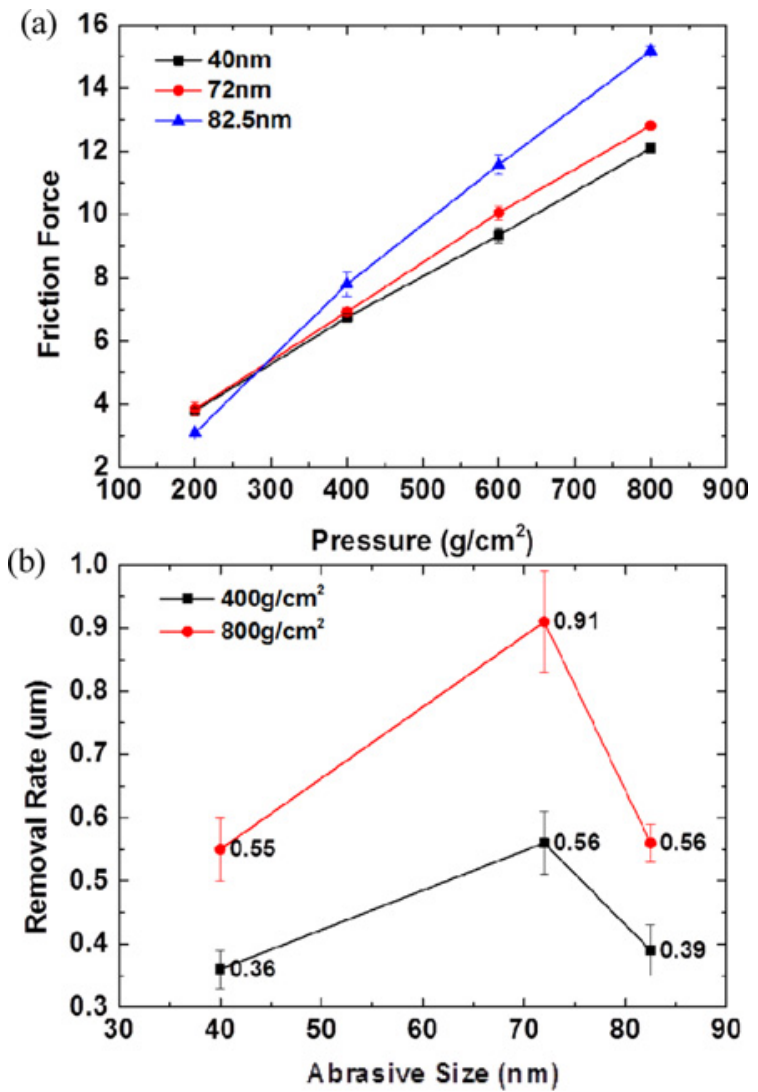

Fig. 4 Effect of abrasive size (with constant number of avrasices) on the (a) Frictional force and (b) Removal rate

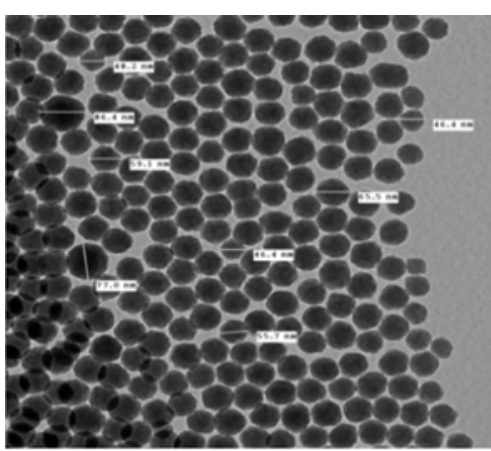

(a) $40 \mathrm{~nm}$

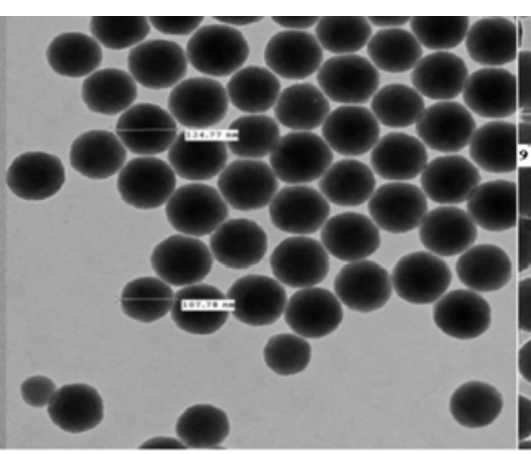

(b) $72 \mathrm{~nm}$

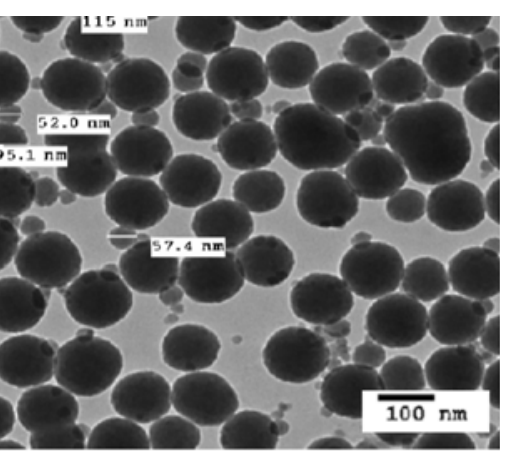

(c) $82.5 \mathrm{~nm}$

Fig. 3 TEM images $(\times 20,000$ magnification) of slurries with the indicated abrasive sizes 
large variation in particle sizes. Since the actual contact area might increase linearly with the pressure, the probability of contact between any given abrasive and the wafer would likewise increase. ${ }^{17}$ As a result, the abrasive size does not show any specific tendency with respect to the pressure.

Lu has similarly found that the removal rate increase when the abrasive size increases. ${ }^{8}$ The current experiment confirms this finding with the added constraint that the number of abrasives per unit volume is constant.

According to indentation theory and contact mechanics, the total removal amount, $\mathrm{V}$, is proportional to the total contact area of the abrasive, $\Delta \mathrm{A}$, the moving distance across the surface, 1 , and the number of active abrasives, $n$ (particles participating in the CMP process); Fig. 5 shows a schematic diagram of these relationships. ${ }^{18}$ For a constant of proportionality, $\mathrm{k}$, this relationship can be expressed by the equation

$$
V=K \cdot(\Delta A \cdot l) \cdot n
$$

The total contact area depends on the indentation depth; when the number of particles increases, the pressure applied per abrasive decreases, ${ }^{19}$ resulting in a shallower depth of indentation. The contact area of each abrasive thus decreases with increasing particle number concentration, along with the volume removed by each.

The principal abrasive factor is then the total contact area of all of the abrasives: $\Delta \mathrm{A} \cdot \mathrm{n}$. The contact area of a single abrasive can be varied by changing the abrasive size. Therefore, an optimal removal rate relies on a combination of the abrasive size and the number of abrasives. Furthermore, the mechanical effect of a particular slurry can be calculated using Eq. (1).

The pressure in CMP alters the contact area and the contact pressure between the pad and substrate. ${ }^{17}$ This, in turn, affects the contact area of the abrasives. Therefore, the removal rate always increases with increasing pressure, regardless of the abrasive size and density, because the specific surface area increases.

The results from the $82.5 \mathrm{~nm}$ slurry show that if the particles are not monodisperse and irregularly shaped, the effect on the removal rate changes dramatically. If the size difference between big and small particles is large, there will generally be more particles participating in the polishing, so the removal rate will increase. However, for the slurry used in this experiment the abrasive size formed a wide distribution, allowing the abrasives to disturb each other and causing some of the abrasives not to participate in the polishing. This manifested as a decrease in the removal rate. ${ }^{20}$

\subsection{Effect of Abrasive Concentration}

In order to understand the dependence of the removal rate on the abrasive concentration, the $40 \mathrm{~nm}$ slurry was diluted to particle number concentrations of $7.9 \times 10^{14} \mathrm{~cm}^{-3}$ at $6 \mathrm{wt} \%$ and $6.9 \times 10^{15} \mathrm{~cm}^{-3}$ at $40 \mathrm{wt} \%$.

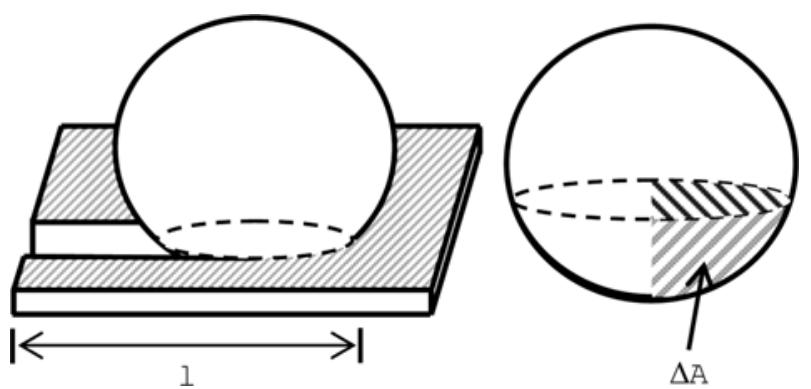

Fig. 5 Schematic diagram of abrasive
Fig. 6 shows that the removal rate increases with increasing concentration of the $40 \mathrm{~nm}$ slurry. These results show that the removal rate always increases with the pressure; this is further related to the abrasive concentration, which makes high concentration slurries useful even in high-pressure CMP.

\subsection{Removal Rate in Different Abrasive Size}

Fig. 7 shows that the removal rate decreases as the abrasive size increases, keeping the weight percent of abrasives in the slurry constant. The number of abrasive particles must therefore increase when the abrasive size decreases; the maximum number of abrasives in slurry is dictated by the particle masses. Although the size and number

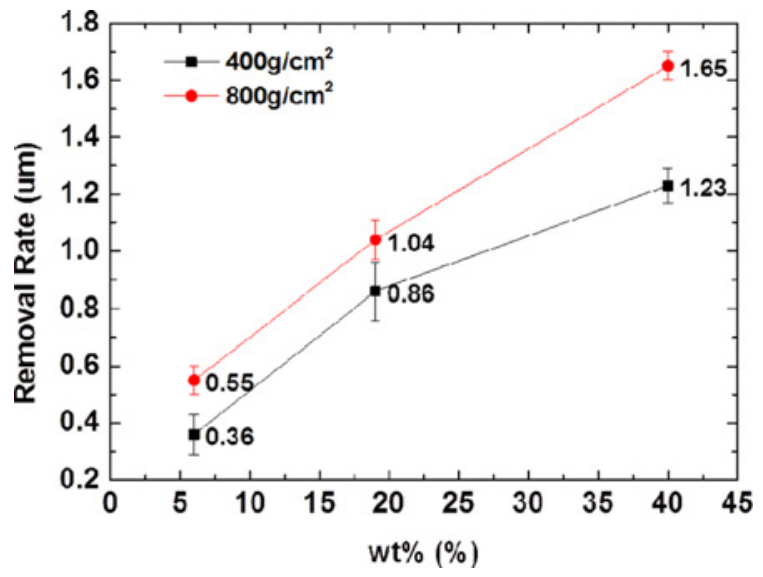

Fig. 6 Remocal rate with weight percent (abrasice size $40 \mathrm{~nm}$ )

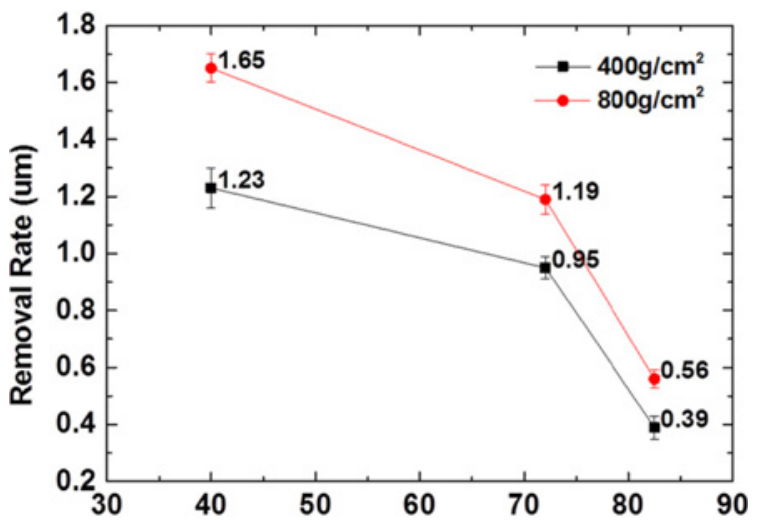

Fig. 7 Removal rate by abrasives with the same seight concentration

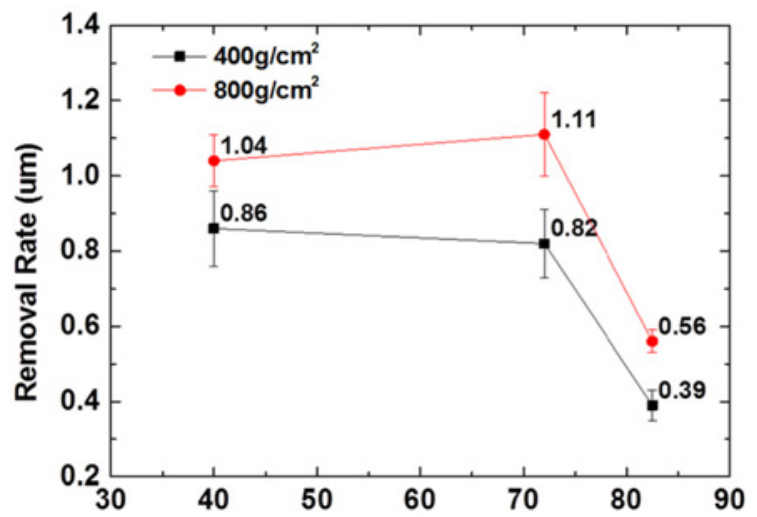

Fig. 8 Removal rate by abrasives with the same specific surface area 
of abrasives are important factors in sapphire wafer CMP, the specific surface area is a convenient parameter because it considers both factors simultaneously. The removal rate is strongly affected by the specific surface area $\left(33 \mathrm{~m}^{2} / \mathrm{g}\right)$, as shown in Fig. 8 . For the $40 \mathrm{~nm}$ and $72 \mathrm{~nm}$ slurries, the removal rate is nearly constant, while the removal rate for the $82.5 \mathrm{~nm}$ slurry decreases significantly. These experiments suggest that the removal rate is strongly affected by the specific surface area.

\section{Conclusions}

1. There has also been a considerable amount of research on the effect of abrasive size in CMP. However, this is the first time that both the abrasive effect in sapphire wafer CMP and the effect of high pressure on the abrasive effect have been studied. The removal rates at various abrasive sizes are found to be similar to other kinds of wafers, e.g., when the abrasive size is large, the removal rate is also high. In this paper, the most important mechanical abrasive factor is found to be the specific surface area; the abrasive size and concentration in the slurry can be selected to optimize the removal rate. This optimum abrasive specific surface area is always same even though pressure is changed. Therefore, optimal pressure for reducing the polishing time for sapphire wafer CMP, and consequently the COC, can be determined regardless of the abrasive size used.

2. Surface roughness is another factor of concern in sapphire wafer CMP because it disturbs the growth of $\mathrm{GaN}$ on the substrate. In highpressure CMP, the surface roughness only increases by a very small amount, but there is the possibility of a scratch forming; if a scratch is not formed, the substrate can be used for epitaxy. However, it is difficult to know whether a scratch is forming during the CMP process and it is not known how to better control for scratch formation,; more research into high-pressure CMP is needed to address these issues. However, it is known that the abrasive size does not influence the surface condition in this experiment.

3. There has been a considerable amount of research on nonmonodisperse and irregular abrasives, but the mechanism acting between the abrasive and the sample is not well understood. Therefore, it would be interesting to further study non-monodisperse and irregular abrasives to gain some insight.

\section{REFERENCES}

1. Niu, X.-H., Liu, Y.-L., Tan, B.-M., Han, L.-Y., and Zhang, J.-X., "Method of Surface Treatment on Sapphire Substrate," Transactions of Nonferrous Metals Society of China, Vol. 16, pp. s732-s734, 2006.

2. Lee, Y.-J., Lin, P.-C., Lu, T.-C., Kuo, H.-C., and Wang, S., "Dichromatic Ingan-Based White Light Emitting Diodes by Using Laser Lift-Off and Wafer-Bonding Schemes," Applied Physics Letters, Vol. 90, No. 16, Paper No. 161115, 2007.

3. Cabalu, J., Bhattacharyya, A., Thomidis, C., Friel, I., Moustakas, T., et al., "High Power Ultraviolet Light Emitting Diodes based on GaN AlGaN Quantum Wells Produced by Molecular Beam Epitaxy," Journal of Applied Physics, Vol. 100, No. 10, Paper No. 104506, 2006.
4. Hu, X., Song, Z., Pan, Z., Liu, W., and Wu, L., "Planarization Machining of Sapphire Wafers with Boron Carbide and Colloidal Silica as Abrasives," Applied Surface Science, Vol. 255, No. 19, pp. 8230-8234, 2009.

5. Murata, J., Kubota, A., Yagi, K., Sano, Y., Hara, H., et al., "Chemical Planarization of Gan using Hydroxyl Radicals Generated on a Catalyst Plate in $\mathrm{H}_{2} \mathrm{O}_{2}$ Solution," Journal of Crystal Growth, Vol. 310, No. 7, pp. 1637-1641, 2008.

6. An, J. H., Lee, G. S., Lee, W. J., Shin, B. C., Seo, J. D., et al., "Effect of Process Parameters on Material Removal Rate in Chemical Mechanical Polishing of 6H-SiC (0001)," Proc. of the Materials Science Forum, pp. 831-834, 2009.

7. Zhu, H., Tessaroto, L. A., Sabia, R., Greenhut, V. A., Smith, M., et al., "Chemical Mechanical Polishing (CMP) Anisotropy in Sapphire," Applied Surface Science, Vol. 236, No. 1, pp. 120-130, 2004.

8. Lu, Z., Lee, S.-H., Babu, S., and Matijeviæ, E., "The Use of Monodispersed Colloids in the Polishing of Copper and Tantalum," Journal of Colloid and Interface Science, Vol. 261, No. 1, pp. 55-64, 2003.

9. Lee, J.-T., Won, J.-K., and Lee, E.-S., "A Study on the Characteristics of a Wafer-Polishing Process according to Machining Conditions," Int. J. Precis. Eng. Manuf., Vo. 10, No. 1, pp. 23-28, 2009.

10. Budnikov, À., Vovk, Å., Krivonogov, S., Danko, À. Y., and Lukiyenko, Î., "Anisotropy of Sapphire Properties Associated with Chemical Mechanical Polishing with Silica," Functional Materials, Vol. 17, No. 4, pp. 489, 2010.

11. Fu, G., Chandra, A., Guha, S., and Subhash, G., “A Plasticity-Based Model of Material Removal in Chemical-Mechanical Polishing (CMP)," IEEE Transactions on Semiconductor Manufacturing, Vol. 14, No. 4, pp. 406-417, 2001

12. Ahmadi, G. and Xia, X., "A Model for Mechanical Wear and Abrasive Particle Adhesion during the Chemical Mechanical Polishing Process," Journal of the Electrochemical Society, Vol. 148, No. 3, pp. G99-G109, 2001.

13. Bielmann, M., Mahajan, U., and Singh, R. K., "Effect of Particle Size during Tungsten Chemical Mechanical Polishing," Electrochemical and Solid-State Letters, Vol. 2, No. 8, pp. 401-403, 1999.

14. Tseng, W.-T., Wang, Y.-L., and Niu, J., "Microstructure-Related Resistivity Change after Chemical-Mechanical Polish of Al and W Thin Films," Thin Solid Films, Vol. 370, No. 1, pp. 96-100, 2000.

15. Lee, H. and Jeong, H., "Analysis of Removal Mechanism on Oxide CMP using Mixed Abrasive Slurry,” Int. J. Precis. Eng. Manuf., Vol. 16, No. 3, pp. 603-607, 2015.

16. Yebing, T., Zhaowei, Z., and Jun, H. N., "Effects of Chemical Slurries on Fixed Abrasive Chemical-Mechanical Polishing of Optical Silicon Substrates,” Int. J. Precis. Eng. Manuf., Vol. 14, No. 8, pp. 1447-1454, 2013.

17. Yu, T.-K., Yu, C., and Orlowski, M., "Combined Asperity Contact and Fluid Flow Model for Chemical-Mechanical Polishing," Proc. 
of the International Workshop on Numerical Modeling of Processes and Devices for Integrated Circuits, NUPAD V., pp. 29-32, 1994.

18. Burwell, J. T., “Wear,” Vol. 1, pp. 119-141, 1957.

19. Ahmadi, G. and Xia, X., "A Model for Mechanical Wear and Abrasive Particle Adhesion during the Chemical Mechanical Polishing Process," Journal of the Electrochemical Society, Vol. 148, No. 3, pp. G99-G109, 2001.

20. Basim, G., Adler, J., Mahajan, U., Singh, R., and Moudgil, B., "Effect of Particle Size of Chemical Mechanical Polishing Slurries for Enhanced Polishing with Minimal Defects," Journal of the Electrochemical Society, Vol. 147, No. 9, pp. 3523-3528, 2000. 